Vol. 5, Issue 10, October 2018

\title{
Modified Training Algorithm for Spiking Neural Network and its Application in Wireless Sensor Network
}

\author{
Mohammed Mousa Rashid Al-Yasari ${ }^{1}$, Nadia Adnan Shiltagh Al-Jamali ${ }^{2}$ \\ Iraqi Commission for Computers and Informatics, Informatics Institute for Postgraduate Studies, Baghdad, Iraq ${ }^{1}$ \\ College of Engineering, University of Baghdad Computer Engineering Department, Baghdad, Iraq ${ }^{2}$
}

\begin{abstract}
In this paper, controlling of data traffic of the Wireless Sensor Network (WSN) is implemented to improve the Quality of Service (QoS) in WSN with mobile sink node. The quality of service is measured in terms of throughput ratio, Packet Loss Ratio (PLR) and Network Energy Consumption (NEC). Spiking neural network is applied to implement the controller that is most suitable with changeable traffic data. The controller is implemented based on python simulation. Simulation results showed that the proposed controller provides better quality of service in comparison with wireless sensor network without controller by about $57 \%$.
\end{abstract}

Keyword: Wireless Sensor Network, Congestion Control in Wireless Sensor Network, improve QoS in Wireless Sensor Network.

\section{INTRODUCTION}

The spread of sensor technology in many regions has gained greater attention to the WSN of the research community and the actual user [1]. WSN is a collection of hundreds or thousands of inexpensive and microscopic sensors, which work together for sensing occurred inside a sensitive area. Each sensor node has the ability to sense as well as the ability to connect and calculate with battery limitations [2]. Each sensor consists of a processor, memory, transceiver, one or more sensors and a battery. WSN is used to monitor events in an area where humans cannot monitor the event [2]. WSN data traffic has distinct characteristics that distinguish it from other traditional networks. The main traffic has a multi-to-one pattern. Most data moves up from the sensor nodes to the base station [3]. Congestion is a common problem in packet transferring networks. Congestion occurs when data sources send data at rates greater than the network's capacity in one or more intermediate methods, which pass through WSN over one or more nodes [4]. New research trends and dissatisfaction solutions have been studied to solve congestion problem in wireless sensor networks. The fundamental truth to be emphasized in most sources is that TCP congestion detection and congestion prevention technology is not suitable for wireless sensor networks because it consumes a lot of resources and is highly aggressive from the point of view of restricted devices and an unstable environment. Therefore, intelligent techniques are needed in wireless sensor networks. Intelligent technology and information technology were discussed from several perspectives. Information resources and access are in an environment necessary for both people and robots. Therefore, the surrounding environment must have sensors organized to collect, store, transform and provide information. Such an environment is called an informational space [5,6]. The rest of this paper is organized as follows. Section 2 explains congestion in WSN. And, Section 3 explains a spiking neural networks. Section 4 the proposed congestion control. Section 5 shows the network design of the proposed method. Finally 6 shows simulation results and conclusion.

\section{CONGESTION IN WSN}

Wireless Sensors Network consists of diverse sensor nodes arrayed in a specific area. These sensor nodes have the ability to get sensitized by the nearby environment and send data which is sensed to the destination sink. Congestion control is a major challenge in WSN [7]. The congestion can occur at any time interval when the total sum of requests on the source is greater than its available capacity. In this case, it is said that there was a congestion in this period of time which is mathematically expressed by Eq. (1)

$\sum$ Demand $>$ Available Resource ..........(1)

Controlling congestion in WSN is a very important critical area of wireless sensors network, where the relatively simple increase in the transmission of data from each node. Since the large number of sensor nodes in the network leads to congestion, the throughput of the network will be reduced [8]. Congestion leads to many drawbacks like increases 


\section{International Advanced Research Journal in Science, Engineering and Technology}

Vol. 5, Issue 10, October 2018

energy waste rates of sensor nodes. A lot of packet loss, leads to reduce the network throughput and prevents dependable data transmission and fair event detections[9]. Large queue delays occur when the packet arrival rate is greater than the link capacity lead to retransmission to recover the dropped packets due to overflow of buffer $[9,10]$.

\section{SPIKING NEURAL NETWORKS [11,12,13,14,15]}

Spiking Neural Networks (SNNs) have the same structure of ANN, but the only two differences between them are the number of synaptic terminals between each layer of neurons and the other difference is the synaptic delay. The feed forward network is used by spike prop in addition to using multiple delayed synaptic terminals. Every neuron can at least create one spike through the stimulation time and can be fired when the membrane potential reaches the threshold value $(\vartheta)$. After all of those events, the neuron will be reset. The network consists of different layers that are named as; $\mathrm{H}$, I, and $\mathrm{J}$ as they shown in Fig. (1). Every single connection among those three layers is composed of group of the same number of synaptic terminals as they symbolized by D letter. Everyone of sub-connection is related with different weight and delay as it is cleared in Fig. (1).

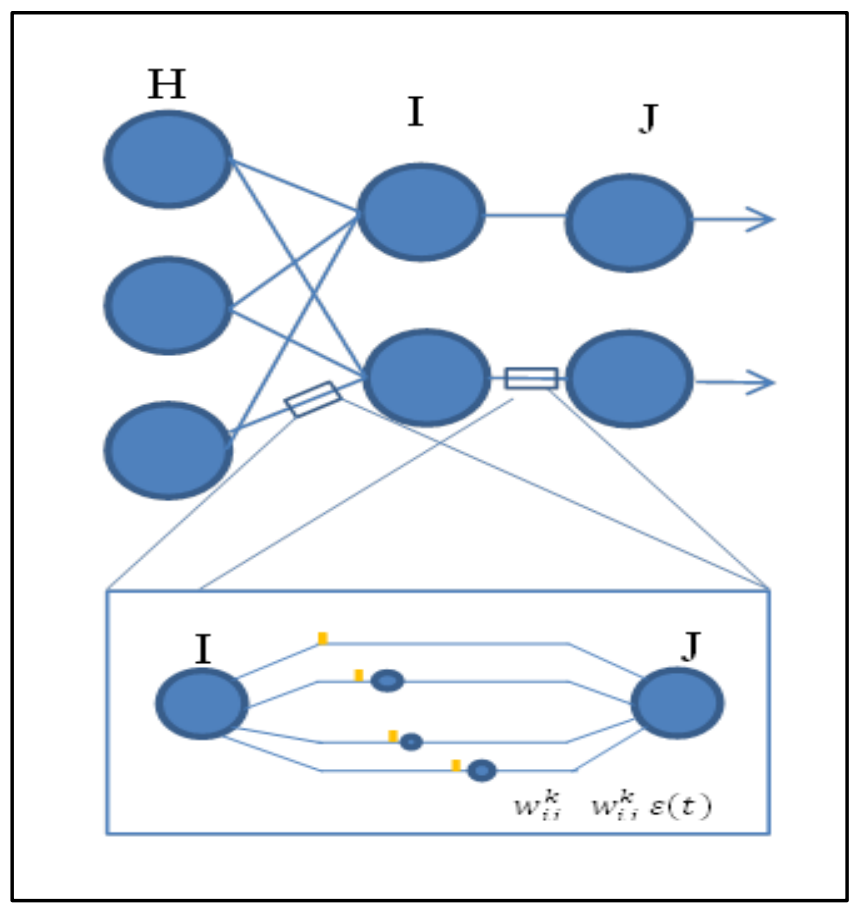

Figure 1: Feed forward Spiking Neural Network

The difference between the time of postsynaptic potential and firing time of presynaptic neurons (i) is defined as the delay of synaptic terminals. The time of postsynaptic potential begins to rise as it cleared in Fig. (2), and there is a synapse sequence in the connection.

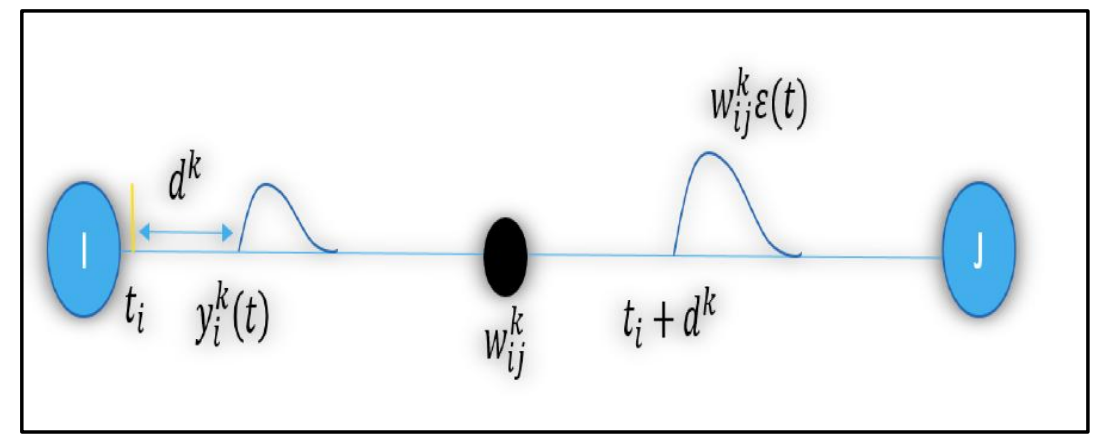

Figure 2: Single synaptic terminal

Where $\mathrm{w}_{\mathrm{ij}}^{\mathrm{k}}$ represent as weight for each sub connection, which remains constant during a simulation, but is updated by Spike Prop afterwards. 


\section{International Advanced Research Journal in Science, Engineering and Technology}

Vol. 5, Issue 10, October 2018

\section{CONGESTION CONTROL IN WSN}

Congestion control in wireless sensors network generally consists of three important components: Congestion Detection Unit (CDU), Congestion Notification Unit (CNU) and Congestion Control Unit (CCU) [16] .

\subsection{Congestion Detection Unit (CDU)}

The congestion detection accurately and efficiently plays a key role in the congestion control in the wireless sensors network, which means identify the possible events that the probability of congestion occurrence is very high [16].

\subsection{Congestion Notification Unit (CNU)}

The transmission of information about congestion occurrence enhances analysis and decision-making as it is essential in effective mechanism of congestion control. There are two types of congestion warning that notify other nodes: explicitly and implicitly [17]. In explicitly type, the nodes which detect congestion, broadcast a control packets to inform some or all member nodes of the network. And implicit notification; does not need additional control messages for propagate congestion information. Piggybacked the congestion information on the normal data packets $[16,17]$.

\subsection{Congestion Control Unit (CCU)}

Congestion control is concerned on obtaining measures from the network in order to (a) avoiding congestion (congestion avoidance approaches) and (b) mitigating congestion (congestion mitigation approaches) and operate with acceptable performance level of the network, when the demand is near to or exceeds the capacity of network resources[17][18][19]. The control of congestion is concerned with efficient using when any load on the network is occurred[20].

\section{THE PROPOSED CONGESTION CONTROLLER}

Using Spiking Neural Network (SNN) to estimate unknown traffic data value that periodically differs with time, depends on the number of active sensors in the network. The variance (Err) between desired and actual buffer occupancy is used to update weights of the SNN using Back Propagation (BP) training algorithm. The online training is used to control the unknown traffic, thus the SNN must learn the behavior of the network and it controls the network. The structure of SNN consists of one input node, the number of hidden layer, number of their neurons in each layer and number of synapses (sub-connections) that are typically chosen empirically. A lot of hidden layers decrease the speed of the training process, and increase network complexity. The structure's sketch of SNN is shown in Fig (3), the activation function of the neurons in hidden layer is tanh. The number of neurons in each of the three layers input, hidden, output layer is 1,7 and 1 respectively. Input neuron of the SNN is assigned to network Traffic (TR) accumulation rate $\mathrm{TR}(\mathrm{t})$; the number of packet arriving to the sink.

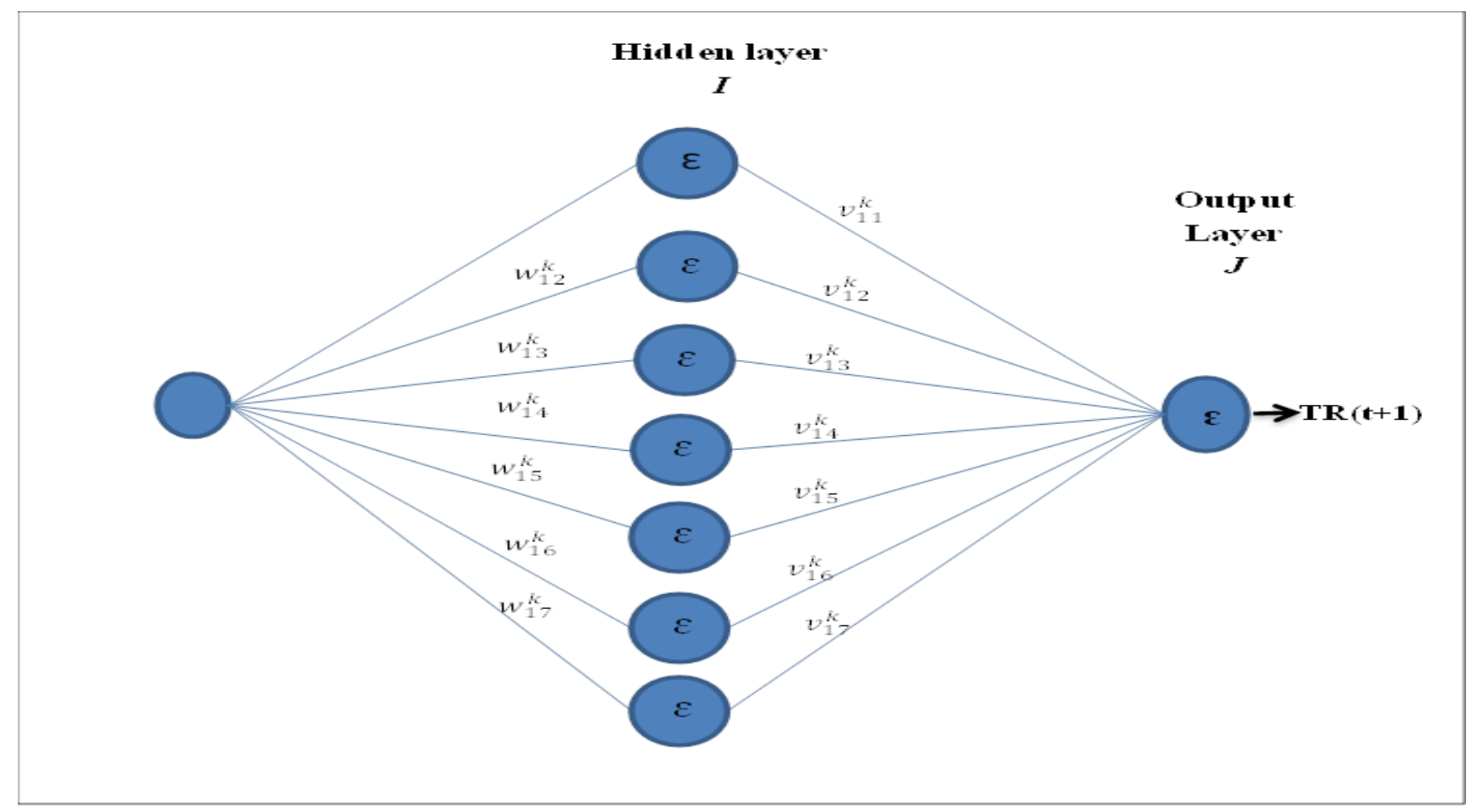

Figure 3: Structure of SNN. 


\section{International Advanced Research Journal in Science, Engineering and Technology}

Vol. 5, Issue 10, October 2018

The number of sub-connection or synapses $\mathrm{k}$ in the relationship between input and hidden layer, and between hidden and output layer, is five delayed sub-connection as shown in Fig. (4). The trial and error method is used to choose the number of synapses $\mathrm{k}$.

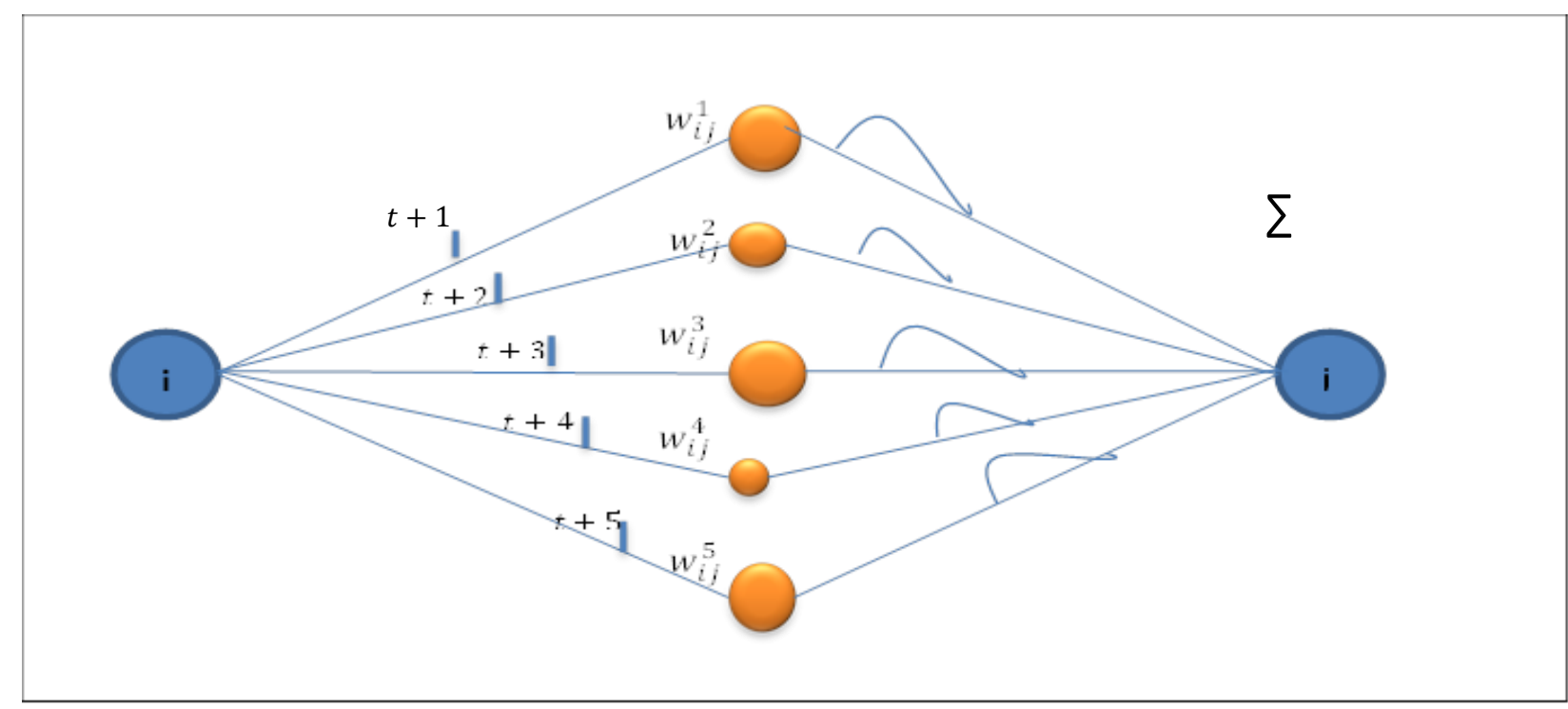

Figure 4: Sub-connection consist of five synapses

As it is clear from Fig. (4), the weight of each synapse $\mathrm{w}_{\mathrm{ij}}^{\mathrm{k}}$ effects on the spike-response function $\varepsilon$; represents the activation function of the neuron.

The TR(t) firstly encoding, in encoding process; the real information TR(t) is encoded information $t_{h}^{\text {act }}$ calculated based on the Eq. (2)

$\mathbf{t}_{\mathbf{h}}^{\text {act }}=\mathbf{t}_{\max }-\operatorname{round}\left(\mathbf{t}_{\min }+\frac{\left(\mathrm{TR}(\mathrm{t})-\mathrm{TR}_{\min }\right)\left(\mathbf{t}_{\max }-\mathbf{t}_{\min }\right)}{\left(\mathrm{TR}_{\max }-\mathrm{TR}_{\min }\right)}\right)$

$\mathrm{TR}_{\max }$ and $\mathrm{TR}_{\min }$ that are represent the maximum and minimum values of the real input information. The $t_{\max }$ and $\mathrm{t}_{\mathrm{min}}$ represent the largest and minimum interval $\mathrm{T}$.

The decoding equation derived from Eq. (2) by Eq. (3)

$\operatorname{TR}\left(t_{\mathbf{j}}\right)=\frac{\left(\mathbf{t}_{\max }-t_{\mathbf{j}}-\mathbf{t}_{\min }\right)\left(\mathbf{T R}_{\max }-\mathbf{T R}_{\min }\right)}{\left(\mathbf{t}_{\max }-\mathbf{t}_{\min }\right)}+\mathbf{T R}_{\min }$

There are two modes for the SNN algorithm. The first is called feed-forward mode where each neuron spikes at each time interval $\mathrm{T}$ only once at most times and that happens when the value $\vartheta$ is exceeded by the membrane potential $\mathrm{m}$. In the feed-forward mode always begins from hidden layer I and examine neuron i continuously to check if it is spiked or not. The algorithm uses the next neuron $\mathrm{i}+1$ when the neuron $\mathrm{i}$ is spiked. The membrane potential $\mathrm{m}_{\mathrm{i}}(\mathrm{t})$ is calculated by the SNN algorithm according to Eq. (4) based on input spikes $t_{h}^{f}$ of neuron $h$ at input layer $H$.

$\mathbf{m}_{\mathbf{i}}(\mathbf{t})=\sum_{\mathbf{h}=\mathbf{1}}^{\mathrm{NH}} \sum_{\mathbf{k}=\mathbf{1}}^{\mathrm{D}} \mathbf{w}_{\mathbf{h i}}^{\mathbf{k}} \varepsilon\left(\mathbf{t}-\mathbf{t}_{\mathbf{h}}^{\mathbf{f}}-\mathbf{d}^{\mathbf{k}}\right)$

Where NH: Number of neurons in input layer and D: Number of delayed-synapses per connection

The neuron i is not allowed to spike anymore through the remaining period of time interval $\mathrm{T}$, when the threshold is exceeded at a particular instant $t$. The neuron $i$ will be reset in next instant $t+1$. The same process will be repeated in the output layer J by the algorithm when the second layer's neurons finished, then the back-propagation phase begins. 


\section{International Advanced Research Journal in Science, Engineering and Technology}

Vol. 5, Issue 10, October 2018

The synapses weights of connection is updated when the feed-forward mode has finished. Opposed to feed-forward the back-propagation begins from output layer and return back to the hidden layer. The synapses of hidden layer will be updated according to Eq. (5).

$\mathbf{w}_{\mathrm{ij}}^{\mathbf{k}}(\mathbf{t}+\mathbf{1})=\mathbf{w}_{\mathrm{ij}}^{\mathbf{k}}(\mathbf{R})-\Delta \mathbf{w}_{\mathrm{ij}}^{\mathbf{k}}(\mathbf{R})$

Eq. (6) and Eq. (7) show how the updating of synapses input layer.

$\Delta \mathbf{w}_{\mathbf{h i}}^{\mathbf{k}}(\mathbf{R})=\eta \cdot \boldsymbol{\delta}_{\mathbf{i}} \cdot \mathbf{y}_{\mathbf{h}}^{\mathbf{k}}$

Where $\eta$ : Learning rate, $\delta_{\mathrm{i}}$ : Delta function.

$\mathbf{w}_{\mathbf{h i}}^{\mathbf{k}}(\mathbf{t}+\mathbf{1})=\mathbf{w}_{\mathbf{h i}}^{\mathbf{k}}(\mathbf{R})-\Delta \mathbf{w}_{\mathbf{h i}}^{\mathbf{k}}(\mathbf{R})$

If the Root Mean Square Error (RMSE) is more than the allowed value of error, the two phases will be repeated.

The output of the SNN is assigned to estimate the network traffic in next time.

The block diagram of the congestion control unit is cleared in Fig. (5).

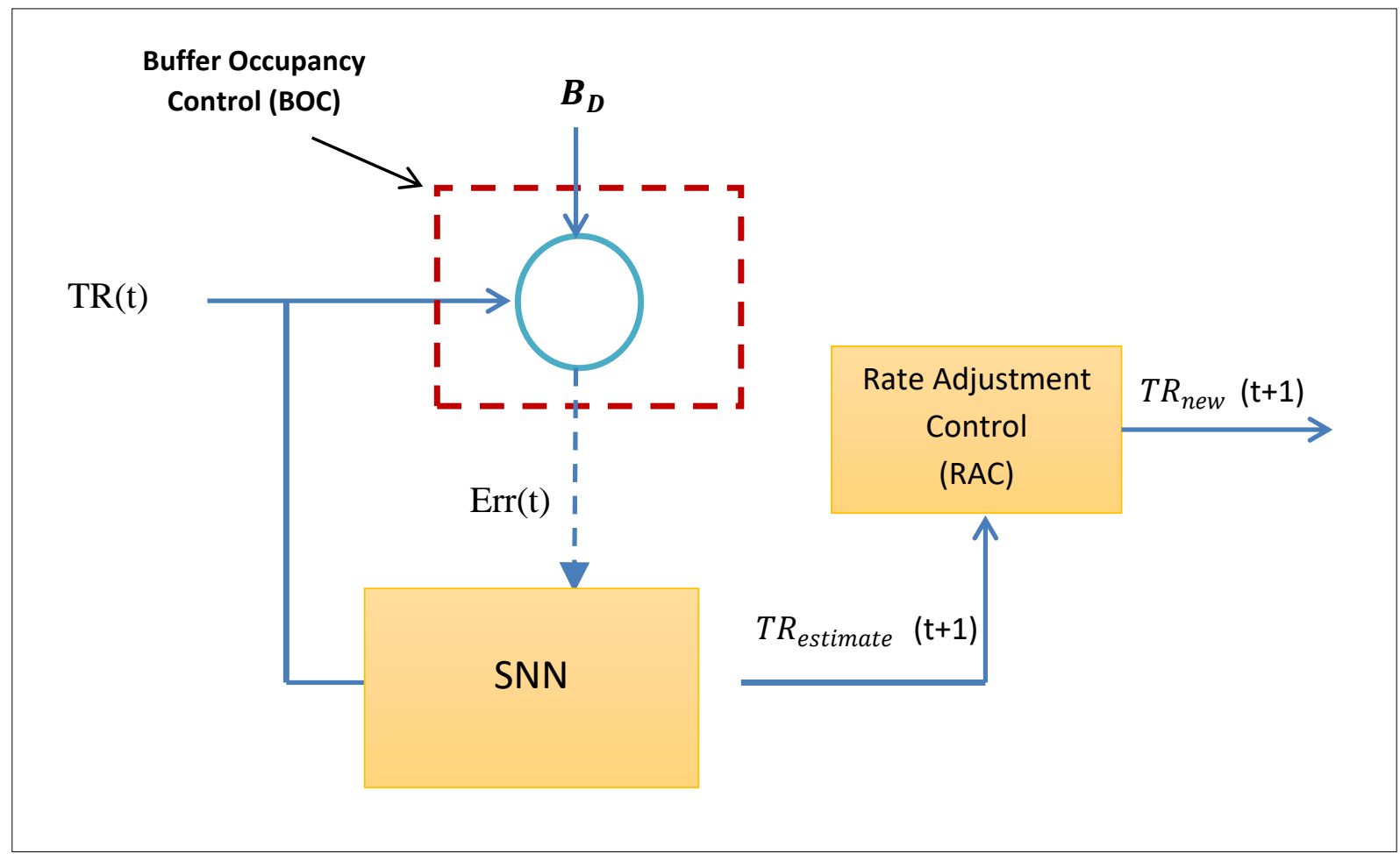

Figure 5: The block diagram of CCU

Fig. (5) shows that the error which can be used to adjust the weights in SNN can be described in Eq.(3.14) and estimation rate is described by Eq. (8).

$$
\operatorname{Err}(\mathbf{t})=\operatorname{TR}(\mathbf{t})-\mathbf{B}_{\mathbf{D}}
$$

Where $B_{D}$ : desired buffer of sink capacity.

The Estimated Traffic Rate $\mathrm{TR}_{\text {estimate }}$ is found out from Buffer Occupancy Control (BOC) and SNN is then divided among the active Cluster Heads (CHs) proportionally to rate in previous time to make fairness among clusters. The function of the Rate Adjustment Control (RAC) can be explained in Eq. (9). 


\section{International Advanced Research Journal in Science, Engineering and Technology}

Vol. 5, Issue 10, October 2018

$\operatorname{TR}_{\text {new }}\left(\mathbf{C H}_{\mathbf{i}}(\mathbf{t}+\mathbf{1})\right)=\frac{\mathrm{TR}_{\text {estimate }}}{\text { number of active } \mathrm{CH}}$

Where the $\mathrm{TR}_{\text {new }}\left(\mathrm{CH}_{\mathrm{i}}(\mathrm{t}+1)\right)$ is the new TR for each $\mathrm{CH}$, which depends on it, $\mathrm{CH}$ determines the number of active sensors in the cluster to avoid congestion in next time.

The new traffic rate is sent to CNU which is taking charge of informing all active clusters of the new TR. Fig. (3) shows the flowchart of the proposed congestion controller in WSN.

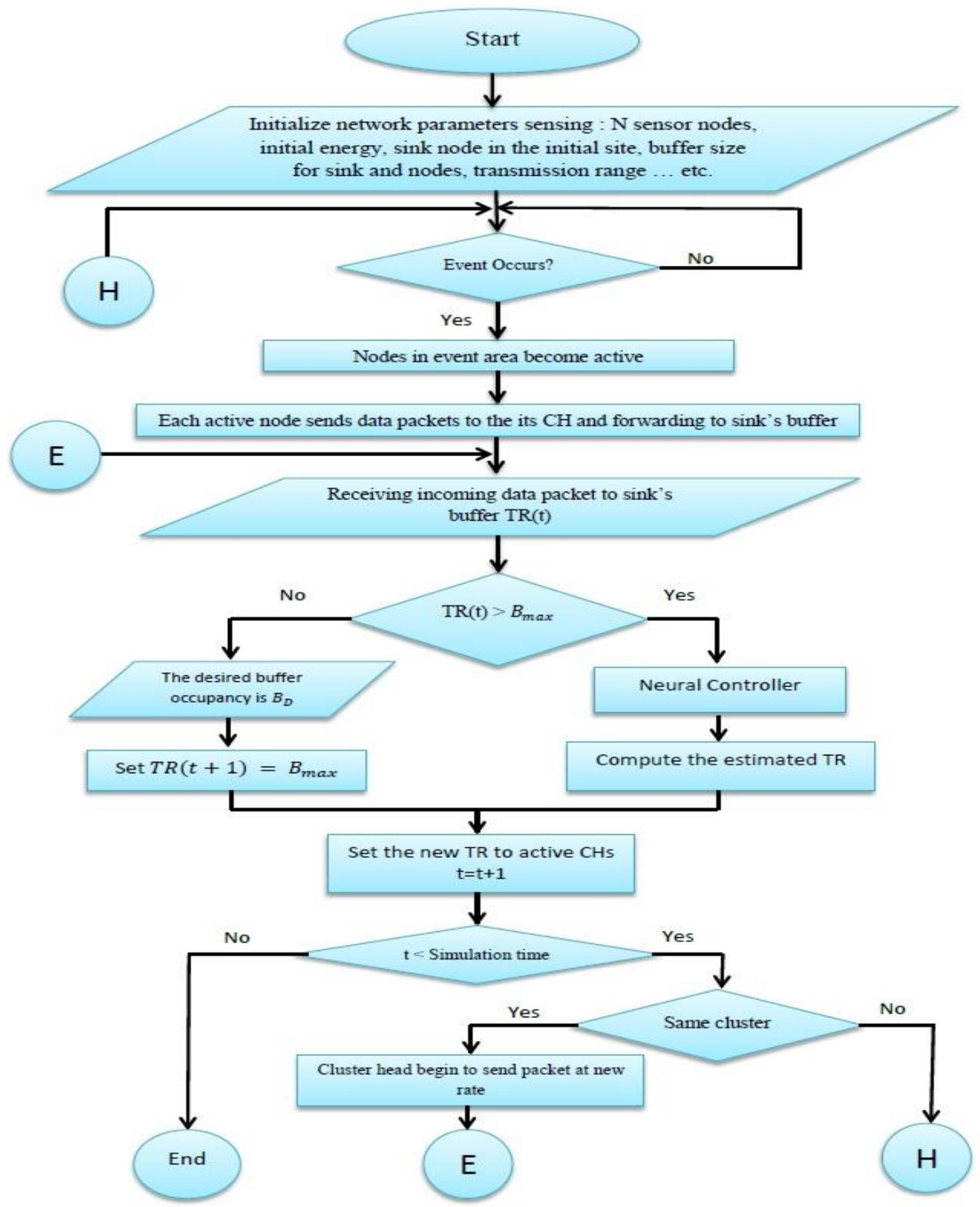

Figure .6: Flow Chart diagram of the proposed congestion controller 


\section{International Advanced Research Journal in Science, Engineering and Technology}

Vol. 5, Issue 10, October 2018

\section{THE NETWORK DESIGN}

The simulation is run with the simulation parameters declared in Table (1). To evaluate the performance under high energy consumption scenario, 100 stationary sensor nodes are randomly placed in (100 meter X 100 meter) with one mobile sink node in the initial location in center of coverage area. Fig.(7) illustrates the model of the 100-nodes in the simulation network. The numbers of active sensors in every cluster are vary depending on the distinct protocol that uses and generates various traffic according to the number of active clusters and active sensors.

Table 1 Simulation parameters

\begin{tabular}{|c|c|}
\hline Parameter & Measurement \\
\hline Ground's dimensions & $100 * 100(\mathrm{~m} * \mathrm{~m})$ \\
\hline Number of nodes & 100 \\
\hline Number of cluster & 4 \\
\hline Node's inner energy & 2 Joule \\
\hline Packet size & 800 byte \\
\hline Sink's buffer size & 250 packets \\
\hline Initial location of the Base Station & 50,50 \\
\hline
\end{tabular}

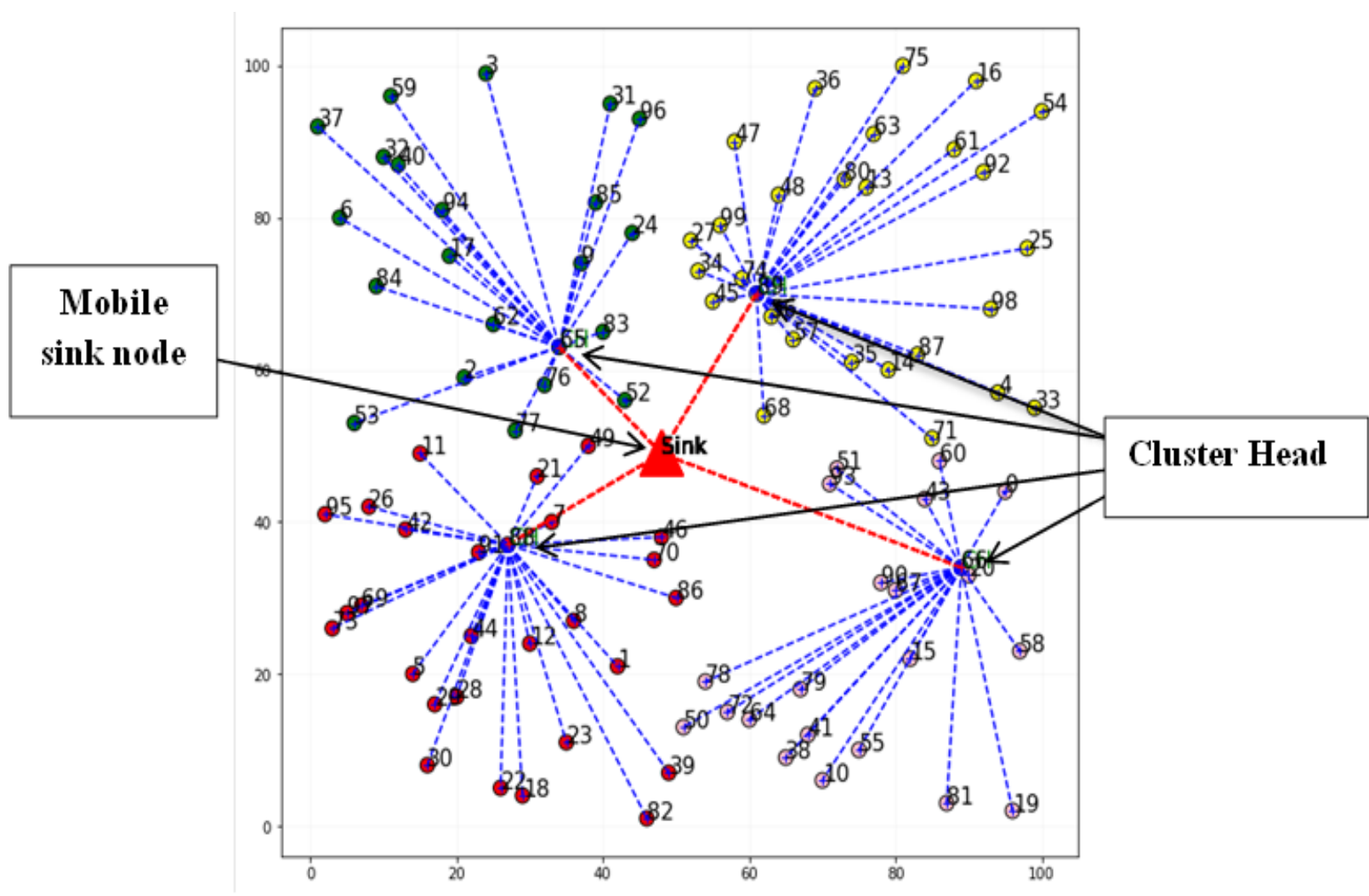

Figure.7: Networking Topology in WSN

\section{SIMULATION RESULTS}

The Spiking Neural Network Congestion Controller (SNNCC), Artificial Neural Network Congestion Controller (ANNCC) are designed in the mobile sink node, the performance metrics are measured, such as the throughput ratio, PLR, network energy consumption and the Network Life Time (NLT), and compared with network Without Congestion Controller (WCC).

Fig. (8) displays throughput ratio of the network, this figure shows that maximize throughput ratio of the network is achieved using congestion controller in comparison with WCC, this feature is more benefit and it is achieved by decreasing traffic via makes coordination between active sensor and maximum capacity of buffer in sink node to send suitable amount of packets from sensors to the available buffer of the network that lead to reduce packets drop causing 


\section{International Advanced Research Journal in Science, Engineering and Technology}

Vol. 5, Issue 10, October 2018

by over load on limited buffer's sink node. It is also obvious that ANNCC performs well but it hasn't accuracy like SNNCC. The performance SNNSRCC is very good.

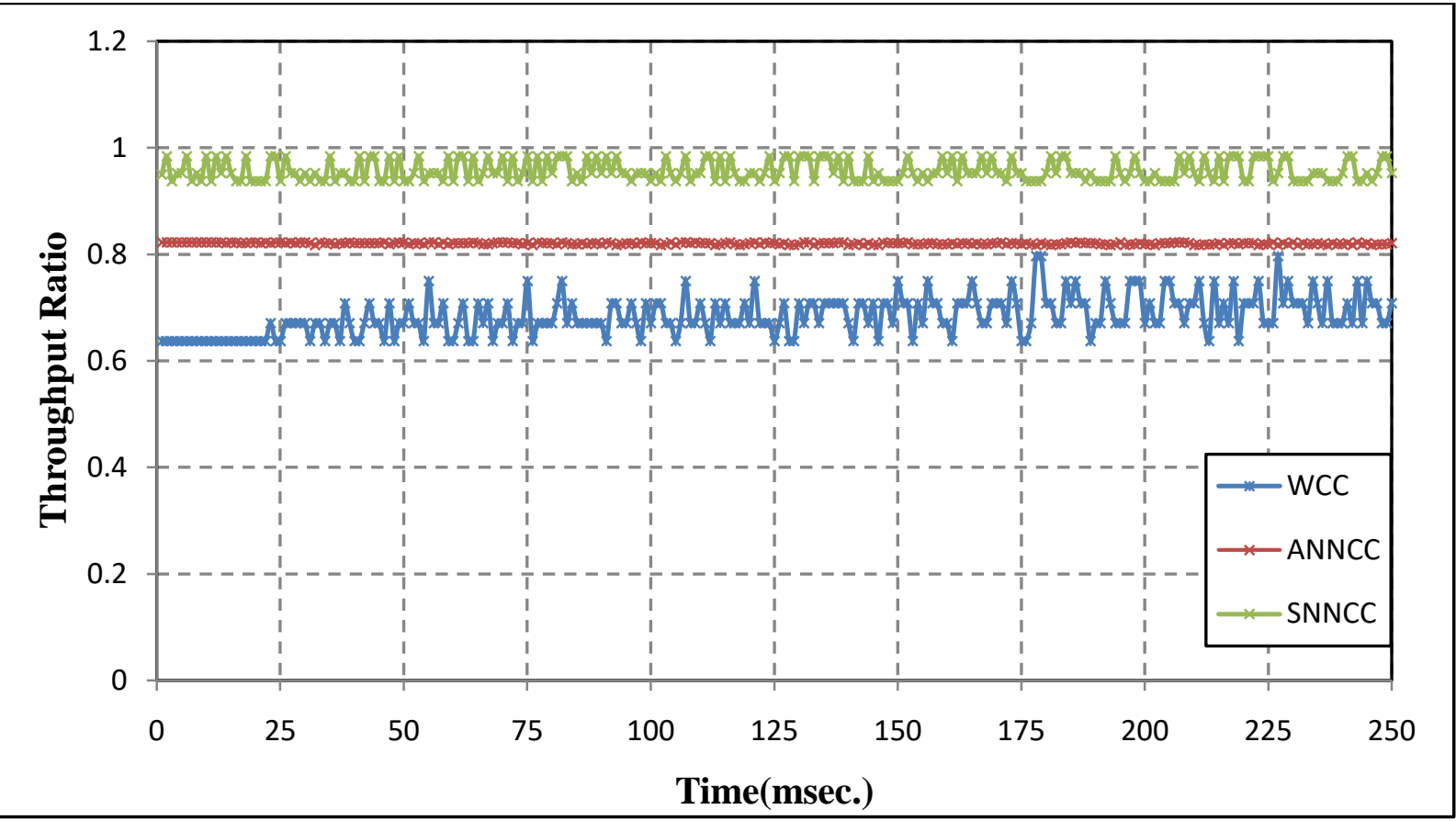

Figure 8: Comparison throughput ratio of network when two proposed congestion controller are used and without

PLR was reduced in congestion controller, therefore the buffer overflow will be reduced in buffer of sink node by sending suitable amount of packets from sensors to the available buffer via making sensor nodes that is out of buffer of sink capacity change to inactive mode (sleep), that is clearly noticed in Fig. 9. Clearly, SNNCC and ANNCC perform well but the accuracy is less when it is compared with SNNCC, which has a high performance.

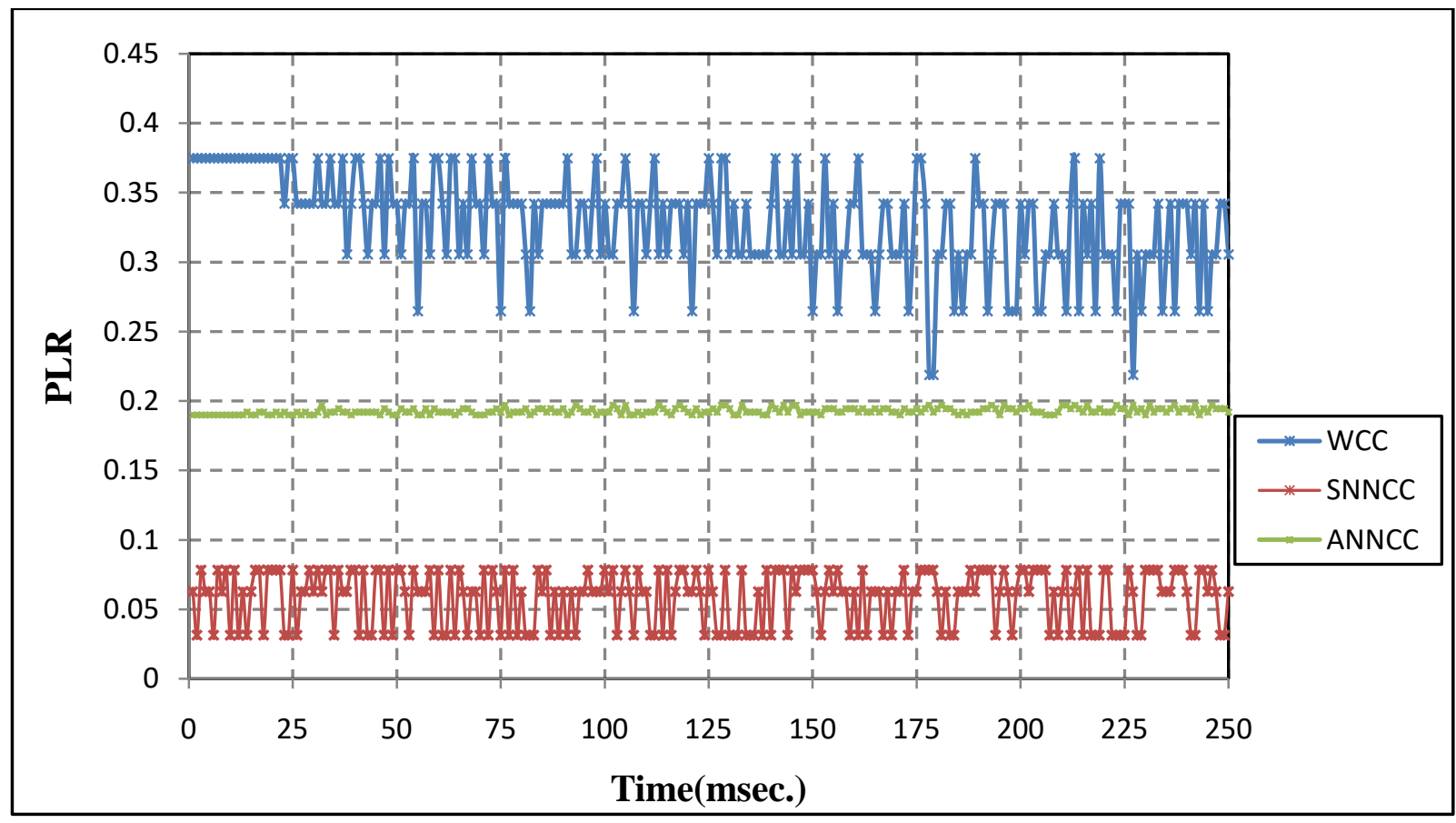

Figure 9: Comparison PLR of network when two proposed congestion controller are used and without 


\section{International Advanced Research Journal in Science, Engineering and Technology}

Vol. 5, Issue 10, October 2018

The increasing of throughput ratio and the decreasing PLR, will decrease the energy consumption because the dissipated energy for sending packets over the buffer capacity which is reduced. It is clear from Fig. 10 which show that EC, the SNNCC save power and prolong life time to about 1100 round time as represented in Fig. 11.

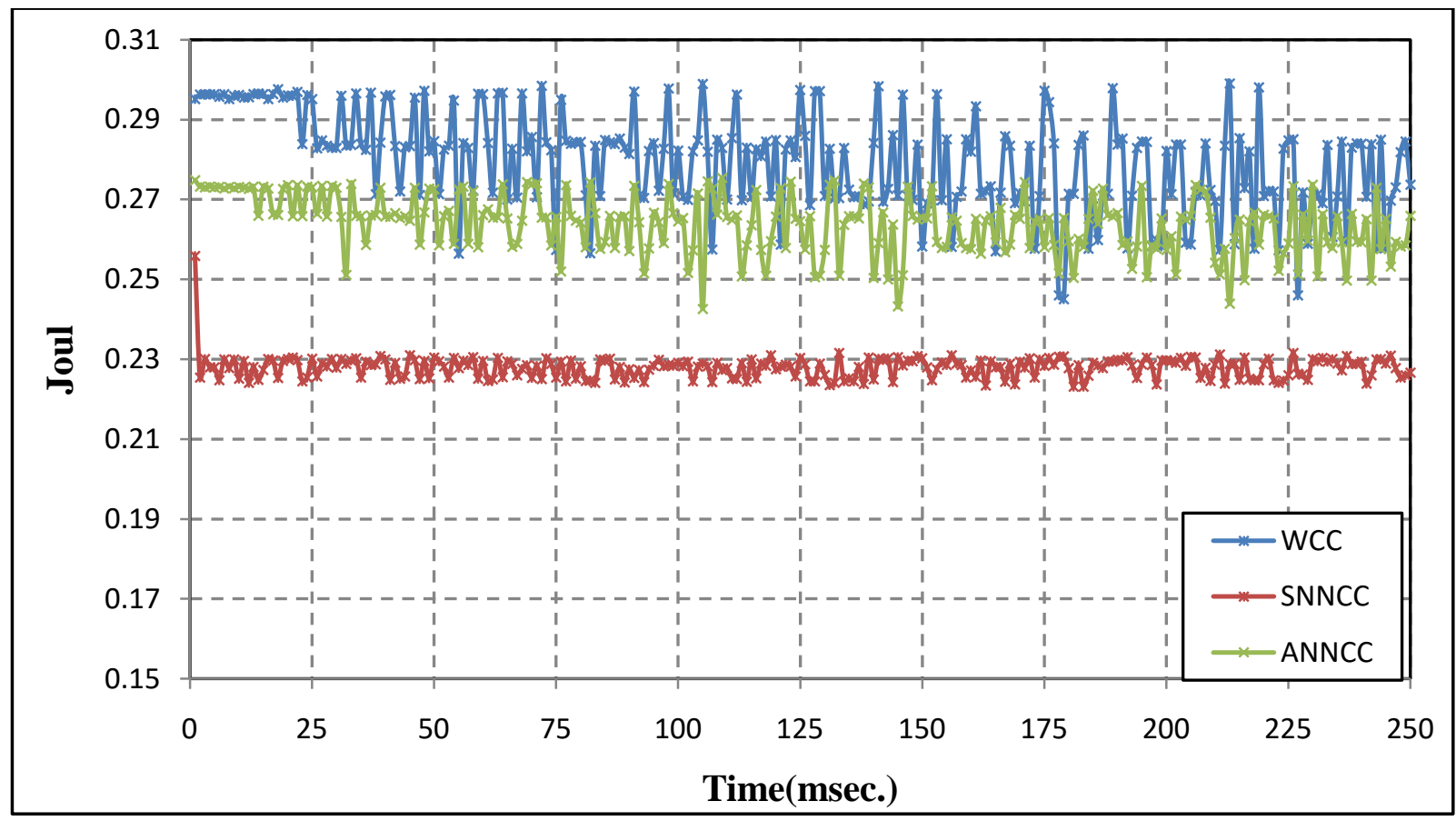

Figure10: Comparison Energy Consumption of network when two proposed congestion controller are used and without

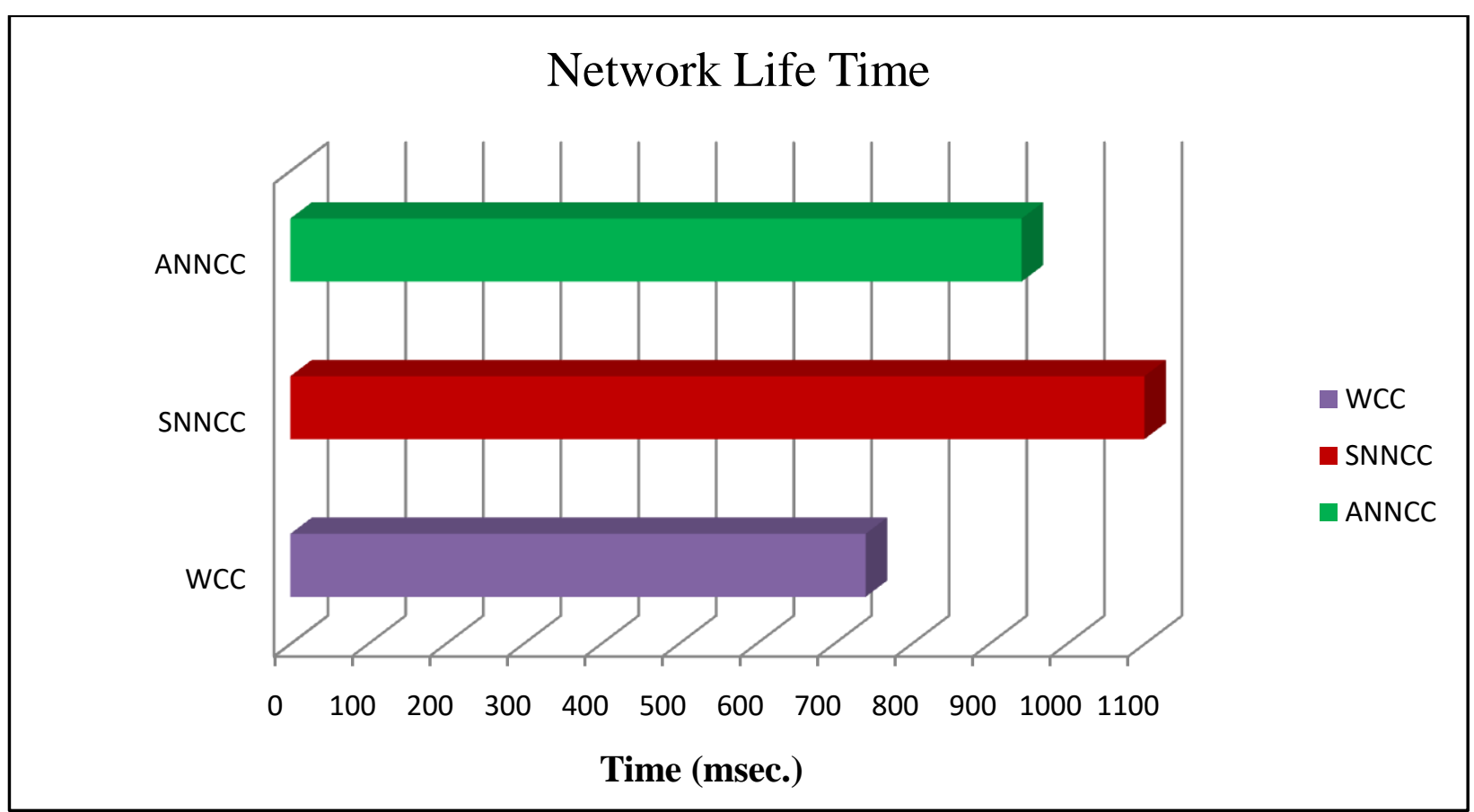

Figure 11: Comparison NLT when two proposed congestion controller are used and without 


\title{
International Advanced Research Journal in Science, Engineering and Technology
}

\author{
Vol. 5, Issue 10, October 2018
}

\section{CONCLUSION}

This paper presented the approach to enhance the QoS in WSN by controlling traffic data to avoid congestion in buffer of mobile sink node based on artificial neural network. The QoS in terms of throughput, Packet Loss Ratio (PLR), Network Energy Consumption (NEC) and network life time. The simulation results and discussion show that the throughput is more increased in WSN that using SNNCC that is estimated new traffic based on SNN; that proved it the most accurate than traditional ANN to estimate a new traffic, and PLR is more reduced than the PLR when using ANNCC and without using congestion controller, reducing the PLR means minimize dissipated energy ,which in turn saving network energy and prolong network life time when using SNNCC.

\section{REFERENCES}

[1] N. F. Ali, A. M. Said, K. Nisar, \& I. A. Aziz, A survey on software defined network approaches for achieving energy efficiency in wireless sensor network, IEEE Conference on Wireless Sensors (2017) 1-6.

[2] M. Song, \& M. Zheng, Energy Efficiency Optimization for Wireless Powered Sensor Networks with Non-orthogonal Multiple Access, IEEE Sensors Letters, 2 (2018) 1-4

[3] M. Koç, \& I. Korpeoglu, Controlled sink mobility algorithms for wireless sensor networks, International Journal of Distributed Sensor Networks, 10 (2014) 1-12.

[4] P. Singhal, \& A. Yadav, Congestion detection in Wireless sensor network using neural network, In Convergence of Technology (I2CT), (2014) $1-4$.

[5] A. G. Elrahim, H. A. , Elsayed, S. El Ramly, \& M. M. Ibrahim, Improving TCP congestion control for wireless sensor networks, In Fly by Wireless Workshop (FBW), (2011) 1-6.

[6] N. Kaur, \& S. Monga, Comparisons of wired and wireless networks: A review, International Journal of Advanced Engineering Technology, 5 (2014) 34-35

[7] G. S. Vyas, \& V. S. Deshpande, Performance of Congestion in Wireless Sensor Network Using Redundant Nodes, In Cloud \& Ubiquitous Computing \& Emerging Technologies (CUBE), (2013) 73-76.

[8] A. Ghaffari, Congestion control mechanisms in wireless sensor networks: A survey,. Journal of network and computer applications, 52 (2015) $101-115$.

[9] M. S. Manshahia, M. Dave, \& S. B. Singh, Bio inspired congestion control mechanism for Wireless Sensor Networks, In Computational Intelligence and Computing Research (ICCIC), (2015) 1-6.

[10] R. M. Kittali, S. K. Mahabaleshwar, \& A. V. Sutagundar, Congestion controlled adaptive routing in wireless sensor network, In Signal Processing, Communication, Power and Embedded System (SCOPES), (2016) 1528-1532.

[11] Y. Oniz, O. Kaynak, \& R. Abiyev, Spiking Neural Networks for the control of a servo system, In Mechatronics (ICM), (2013) 94-98.

[12] A. J. Hussain, \& H. Tawfik, A Spiking Neural Network for Financial Prediction, Proceedings of International Joint Conference on Neural Networks, Dallas, Texas, USA, (2013) 3111-3118

[13] J. Vreeken, Spiking neural networks, an introduction, Ad aptive Intelligence Laboratory, Intelligent Systems Group, (2003) 1-5.

[14] S. M. Bohte, J. N. Kok, \& H. La Poutre, Error-backpropagation in temporally encoded networks of spiking neurons, Neurocomputing, 48 (2002) 17-37.

[15] V. Thiruvarudchelvan, J. W. Crane, \& T. Bossomaier, Analysis of SpikeProp convergence with alternative spike response functions, In Foundations of Computational Intelligence (FOCI), (2013) 98-105.

[16] R. Jan, Congestion Control in Wireless Sensor Networks-An overview of Current Trends, International Journal of Science and Engineering Applications, 5 (2016) 273-279.

[17] S. Patel, R. Pandit, \& M. A. Rathod, Congestion Detection \& Minimization in Wireless Sensor Network By Using Multipath Rate Organization Technique, 3 (2014) $569-575$

[18] M. J. A. Jude, \& V. C. Diniesh, DACC: Dynamic agile congestion control scheme for effective multiple traffic wireless sensor networks, In Wireless Communications, Signal Processing and Networking (WiSPNET), (2017) 1329-1333.

[19] A. A. Khan, S. Ghani, \& S. Siddiqui, Design \& implementation of distributed congestion control scheme for heterogeneous traffic in wireless sensor networks, In Multisensor Fusion and Integration for Intelligent Systems (MFI), (2017) 581-585.

[20] M. A. Kafi, J. Ben-Othman, A. Ouadjaout, M. Bagaa, \& N. Badache, REFIACC: Reliable, efficient, fair and interference-aware congestion control protocol for wireless sensor networks, 101 (2017) 1-11.

[21] S. A. Ahson, \& M. Ilyas, RFID handbook: applications, technology, security, and privacy, CRC press, NewYork, 2008. 